\title{
Nanoscale
}

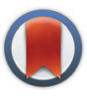

CrossMark $\leftarrow$ click for updates

Cite this: Nanoscale, 2016, 8, 2804

Received 21st October 2015

Accepted 5th January 2016

DOI: 10.1039/c5nr07307c

www.rsc.org/nanoscale

\section{Versatile method for template-free synthesis of single crystalline metal and metal alloy nanowires $\uparrow$}

\author{
John A. Scott, Daniel Totonjian, Aiden A. Martin, $\$$ Toan Trong Tran, Jinghua Fang, \\ Milos Toth, Andrew M. McDonagh, Igor Aharonovich and Charlene J. Lobo*
}

\begin{abstract}
Metal and metal alloy nanowires have applications ranging from spintronics to drug delivery, but high quality, high density single crystalline materials have been surprisingly difficult to fabricate. Here we report a versatile, template-free, self-assembly method for fabrication of single crystalline metal and metal alloy nanowires (Co, $\mathrm{Ni}, \mathrm{NiCo}, \mathrm{CoFe}$, and $\mathrm{NiFe}$ ) by reduction of metal nitride precursors formed in situ by reaction of metal salts with a nitrogen source. Thiol reduction of the metal nitrides to the metallic phase at $550-600{ }^{\circ} \mathrm{C}$ results in nanowire growth. In this process, sulfur acts as a uniaxial structure-directing agent, passivating the surface of the growing nanowires and preventing radial growth. The versatility of the method is demonstrated by achieving nanowire growth from gas-phase, solution-phase or a combination of gas- and solution-phase precursors. The fabrication method is suited to large-area CVD on a wide range of solid substrates.
\end{abstract}

\section{Introduction}

Metal nanowires are building blocks for realizing new devices with applications in optoelectronics, spintronics,${ }^{1-4}$ biosensing and medicine, ${ }^{5}$ as well as in catalysis, ${ }^{6}$ motors, ${ }^{7}$ and drug delivery. ${ }^{8}$ Metal nanowires also enable fundamental studies of ballistic transport and of the effect of dimensionality on phenomena such as spin and orbital momentum and magnetic anisotropy. ${ }^{9}$ Currently, template electrodeposition is the most commonly used method for producing uniform, highdensity metal nanowires. Recent advances in the electrodeposition technique have made it possible to fabricate single crystalline nanowires from noble and low melting point metals. ${ }^{10,11}$ The method has been extended to the ferromagnetic metals $\mathrm{Co}, \mathrm{Fe}$ and $\mathrm{Ni}$ and their alloys, with control over crystal structure and composition..$^{11-13}$ However, electrodeposition is a cumbersome process that requires fabrication of anodic aluminium oxide, polycarbonate or diblock copolymer templates, metal electrodeposition, and template removal. ${ }^{14-17}$ Electrodeposited growth of single-crystal Ni nanowires has only been achieved for small pore diameters $(50 \mathrm{~nm})$, with TEM characterization indicating considerable surface roughness, ${ }^{18}$ while

School of Mathematical and Physical Sciences, University of Technology, Sydney, P.O. Box 123, Broadway, New South Wales 2007, Australia.

E-mail: charlene.lobo@uts.edu.au

$\dagger$ Electronic supplementary information (ESI) available: Nanowire structural characterization by SEM and HRTEM. See DOI: $10.1039 / \mathrm{c} 5 \mathrm{nr} 07307 \mathrm{c}$

$\$$ Present Address: Lawrence Livermore National Laboratory, Livermore, CA 94550 , USA. cobalt nanowires have high concentrations of stacking faults. ${ }^{19}$ Fracturing of high aspect ratio structures, surface roughening and contamination due to post-deposition template removal processes are common problems of the technique. ${ }^{20}$

Template-free self-assembly of single-crystal cobalt and nickel nanowires has been achieved by epitaxial growth in solution and from the vapor phase, ${ }^{21-24}$ and solvothermal ${ }^{25-27}$ methods. However, low yields and substrate limitations have limited applications of epitaxially-grown nanowires, while solvothermal growth methods typically produce aggregates of low aspect ratio nanowires. To date, no general method of template-free self-assembly of binary or ternary metal alloy nanowires has been reported.

Here we report a versatile and scalable fabrication technique for single crystalline metal and metal alloy nanowires (e.g. Co, Ni, NiCo, CoFe, and NiFe). The method employs reduction of metal nitride precursors formed in situ by reaction of metal salts with a nitrogen source. Nanowires may be grown using gas-phase, solution-phase or a combination of gas- and solution-phase precursors. We also examine the roles of the reactants and intermediate metal phases ${ }^{28}$ in the uniaxial growth mechanism.

\section{Results and discussion}

Precursors and growth conditions used to synthesize cobalt, nickel, iron and alloy nanowires from vapor (v) and solution (s) phase precursors are presented in Table 1 . We demonstrate nanowire growth using four different processes. In Process 1, 
Table 1 Experimental conditions used to prepare Co, $\mathrm{Ni}$ and Fe metal and alloy nanowires

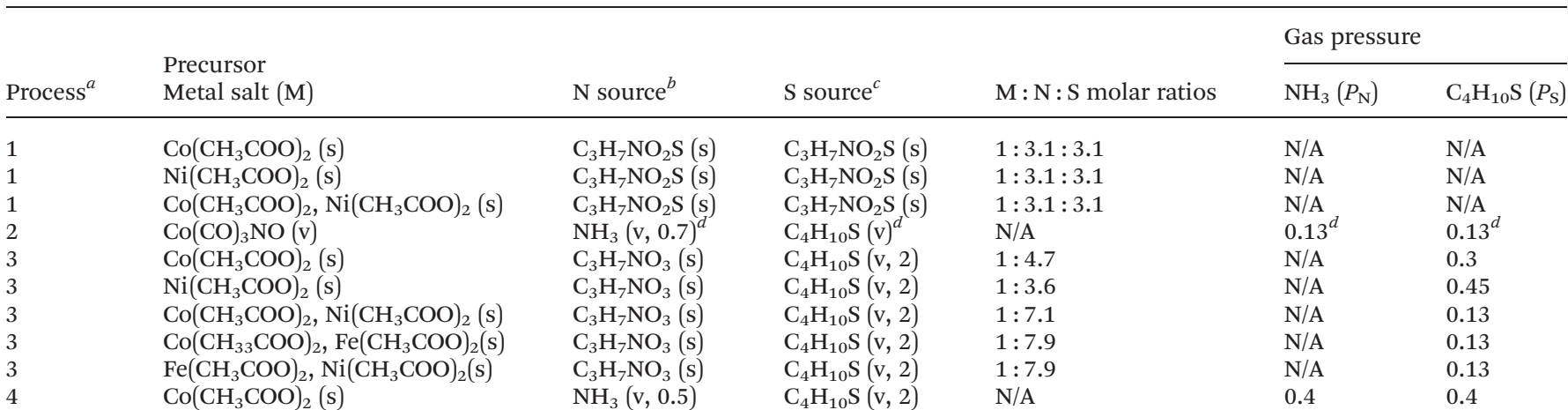

$(\mathrm{s})=$ solid phase precursor. $(\mathrm{v}, \mathrm{sccm})=$ vapor phase precursor, flow rate in sccm. Molar ratios are provided for solution-phase precursors only. Gas pressures have units of mbar. ${ }^{a}$ Annealing was conducted for 1 hour at 550-600 ${ }^{\circ} \mathrm{C}$ in each case. ${ }^{b} \mathrm{C}_{3} \mathrm{H}_{7} \mathrm{NO}_{2} \mathrm{~S}=\mathrm{L}_{\mathrm{L}}-\mathrm{cysteine}, \mathrm{C}_{3} \mathrm{H}_{7} \mathrm{NO}_{3}=\mathrm{L}_{\mathrm{L}}$-serine. ${ }^{c} \mathrm{C}_{3} \mathrm{H}_{7} \mathrm{NO}_{2} \mathrm{~S}=$ L-cysteine, $\mathrm{C}_{4} \mathrm{H}_{10} \mathrm{~S}=$ 1-butanethiol. ${ }^{d}$ Gases mixed before introduction into vacuum chamber.

cobalt nanowires were grown using a single-step annealing process in which an aqueous solution of cobalt(II) acetate, $\mathrm{Co}\left(\mathrm{CH}_{3} \mathrm{COO}\right)_{2}$, and L-cysteine was drop-cast onto a silicon substrate, dried, and then heated under high vacuum $\left(10^{-5} \mathrm{mbar}\right)$ at $600{ }^{\circ} \mathrm{C}$ (Fig. 1a).

The resultant nanowires were characterized by transmission electron microscopy (TEM), energy filtered TEM (EFTEM) and electron energy loss spectroscopy (EELS).

Cobalt, sulfur and carbon EFTEM elemental maps show that the nanowires contain Co and S (ESI Fig. S1a†). Scanning electron microscopy (SEM) reveals that nanowire growth occurs predominately on regions where the dropcast film has delaminated from the substrate. A representative SEM image of a high-yield cobalt nanowire region is shown in Fig. 1b. The nanowires have lengths of up to tens of micrometers and diameters ranging from of 40 to $120 \mathrm{~nm}$ (Fig. 1c). Previous work has established that the only $\mathrm{Co}-\mathrm{S}$ phases that are stable under our growth conditions $(T<650 \mathrm{C}$ and low sulfur concentrations $\left.\left(X_{\mathrm{S}}<0.3\right)\right)$ are the bulk $\mathrm{Co}_{8} \mathrm{~S}_{9}$ phase and Co metal. ${ }^{29}$

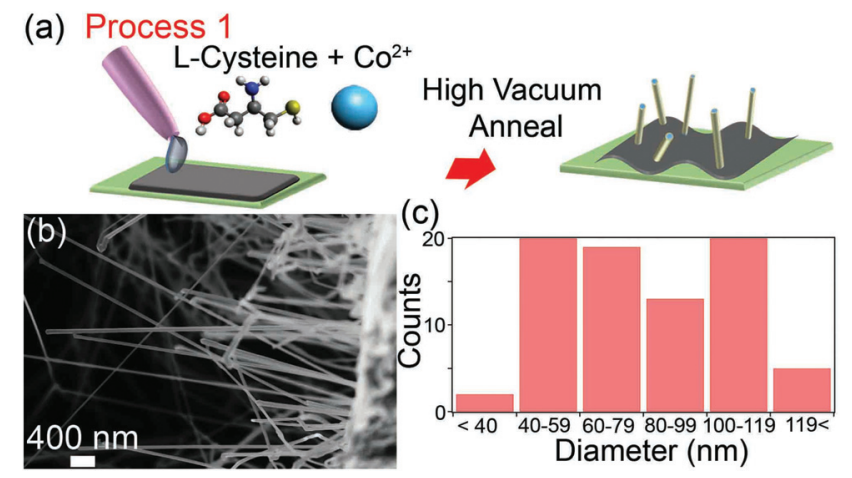

Fig. 1 (a) Schematic of Process 1 showing self-assembly of Co nanowires from a dropcast and dried aqueous precursor solution which was annealed in high vacuum at $600{ }^{\circ} \mathrm{C}$ for $40-60$ minutes. (b) SEM image of the nanowires. (c) Nanowire diameter distribution.
Together, the information obtained from HRTEM and EELS conclusively establishes that the nanowires are single-crystalline cobalt metal, rather than $\mathrm{Co}_{8} \mathrm{~S}_{9}$. EELS spectra (ESI Fig. S1b†) do not contain the oxygen-K edge (at $\sim 540 \mathrm{eV}$ ) that is characteristic of cobalt oxides $\left(\mathrm{CoO}\right.$ and $\left.\mathrm{Co}_{3} \mathrm{O}_{4}\right) \cdot{ }^{30}$ Highresolution TEM images (ESI Fig. 1c $\dagger$ ) revealed that the nanowires are single crystals with lattice fringe-resolved $d$-spacings of 2.02 and $2.03 \AA$ corresponding to the (11-1) planes of face centered cubic (FCC) Co $(2.05 \AA)$, coated with a thin $(\sim 4 \mathrm{~nm})$ amorphous overlayer (for comparison, the lattice spacing of fcc $\mathrm{Co}_{8} \mathrm{~S}_{9}$ is $10 \AA$ A). ${ }^{31}$ Fast Fourier transforms (FFT) (ESI Fig. S1c†) of nanowires grown directly on a gold TEM mesh (ESI Fig. 1d †) confirm that the crystal structure is cubic, and that the growth direction is [11-1]. The presence of Co and S, and the absence of $\mathrm{O}$ and $\mathrm{C}$ in the nanostructures indicate that the amorphous layer at the nanowire surface is composed of sulfur.

To elucidate the roles of each chemical component in the nanowire formation mechanism, Process 1 was repeated using aqueous solutions of cobalt(II) acetate alone (ESI Fig. S2a†), cobalt(II) acetate and the amino acid L-serine which contains no thiol group $\left(\mathrm{C}_{3} \mathrm{H}_{7} \mathrm{NO}_{3}\right.$, ESI Fig. S2b $\left.\dagger\right)$, and cobalt(II) acetate and L-cysteine which contains both amine and thiol groups $\left(\mathrm{C}_{3} \mathrm{H}_{7} \mathrm{NO}_{2} \mathrm{~S}\right.$, ESI Fig. S2c $\left.\dagger\right)$. Cobalt nanowire growth was observed only in the third experiment, demonstrating that nanowire formation requires the presence of both amine and thiol groups in the precursor mixture. Nickel nanowire growth using Process 1 also occurs only when both amine and thiol groups are present (ESI Fig. S2d-f $f$ ). We hypothesized that the role of the amine is to form a metal nitride precursor, which is subsequently reduced by the thermal treatment and/or the thiol during growth to yield the metallic nanostructures. In Process 1, L-cysteine provides a convenient source of both of these moieties in a single molecule.

If the above hypothesis is correct, an all-vapor phase synthesis approach could be envisaged. Thus, L-cysteine was substituted with a combination of gaseous ammonia $\left(\mathrm{NH}_{3}\right)$ and 
(a) Process 2 40-60 minutes.

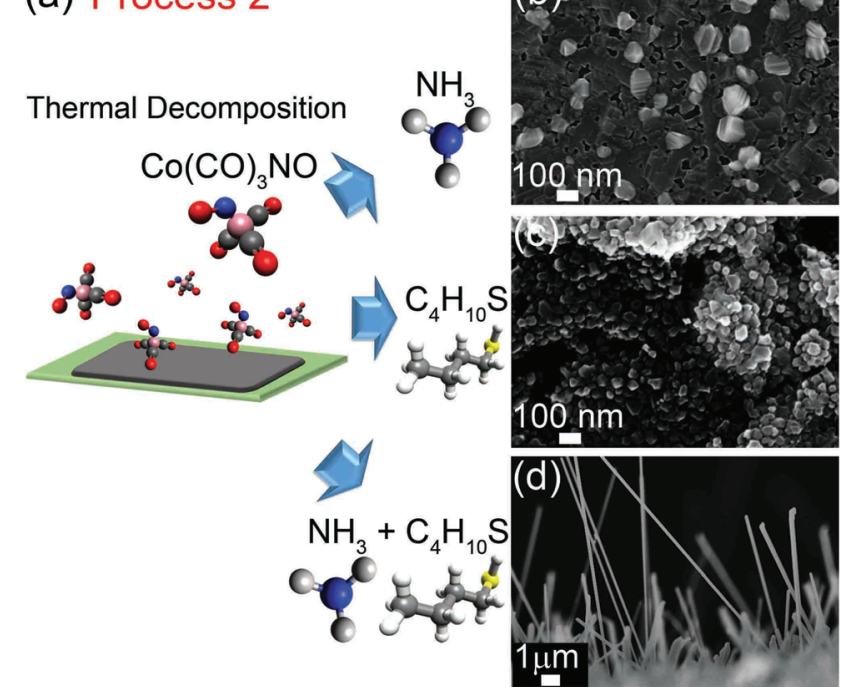

Fig. 2 (a) Schematic illustration of three variants of Process 2. SEM images of nanostructures grown by annealing a film formed by decomposition of $\mathrm{Co}(\mathrm{CO})_{3} \mathrm{NO}$ in (b) gaseous ammonia, (c) butanethiol, and (d) ammonia and butanethiol environments at $550-600{ }^{\circ} \mathrm{C}$ for

1-butanethiol $\left(\mathrm{C}_{4} \mathrm{H}_{10} \mathrm{~S}\right)$ in Process 2 (Fig. 2a). First, cobalt tricarbonyl nitrosyl vapor $\left(0.27\right.$ mbar $\left.\mathrm{Co}(\mathrm{CO})_{3} \mathrm{NO}\right)$ was decomposed onto a silicon substrate at $150{ }^{\circ} \mathrm{C}$. After evacuation of residual $\mathrm{Co}(\mathrm{CO})_{3} \mathrm{NO}$ from the vacuum chamber, the substrate was heated to 550-600 ${ }^{\circ} \mathrm{C}$ and then annealed in either ammonia vapor (Fig. 2b), 1-butanethiol vapor (Fig. 2c) or a mixture of ammonia and butanethiol vapors (Fig. 2d) for 40-60 minutes. When only ammonia or only 1-butanethiol was used, the resultant cobalt nanostructures were irregular in shape and nanowires were absent. In the presence of both ammonia and 1-butanethiol, cobalt nanowires were produced. This experiment confirms our hypothesis that a nitrogen source and a reducing thiol compound are both required for nanowire growth and, importantly, demonstrates that all reactants can be supplied from the vapor phase in a low-pressure, low-temperature CVD growth process.

To further clarify the roles of amines and thiols in the nanowire growth process, we conducted experiments using two-step processes in which these species are introduced separately. In the first variant of this method, $\mathrm{N}$ is incorporated in the liquid phase precursor mixture (Process 3). In the second variant, $\mathrm{N}$ is supplied from the gas phase during an initial annealing step, followed by evacuation of the chamber (Process 4). In both processes, sulfur is then supplied from the gas phase during a second thermal annealing step.

In Process 3 (Fig. 3a), a dropcast aqueous solution of cobalt(II) acetate and L-serine was annealed in a gaseous 1-butanethiol environment at $550{ }^{\circ} \mathrm{C}$ for $1 \mathrm{~h}$ producing cobalt nanowires as shown in Fig. 3b. Annealing cobalt(II) acetate and L-serine in ammonia (Fig. 3c) or in vacuum (ESI Fig. S2b $\dagger$ ) yielded irregular, three dimensional nanocrystallites rather (a) Process $3 \curvearrowright$ L-serine $+\mathrm{Co}^{2+}$

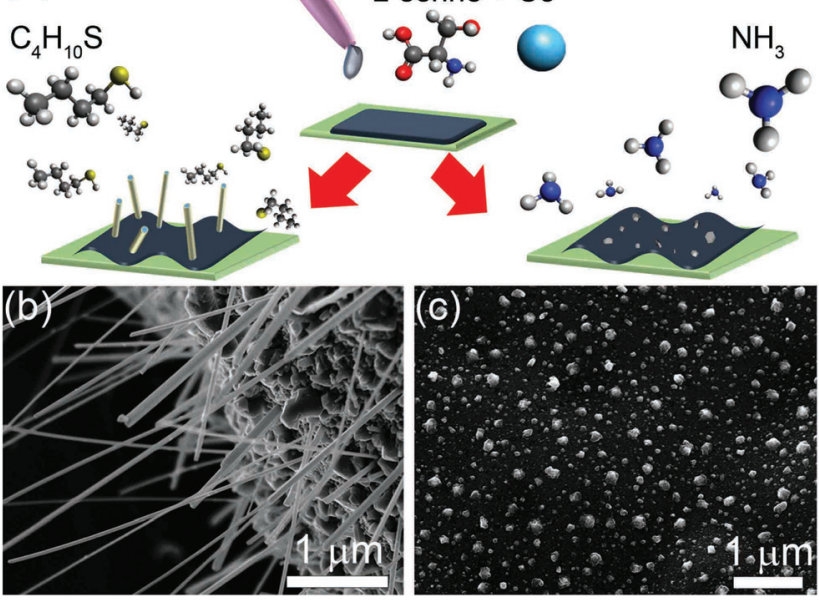

Fig. 3 (a) Schematic of Process 3. SEM images of cobalt nanostructures grown by annealing of drop-cast, dried aqueous solutions of Co(II) acetate and L-serine in (b) butanethiol vapor and (c) ammonia vapor at $550^{\circ} \mathrm{C}$ for $1 \mathrm{~h}$.

than nanowires. Similar results were obtained for Ni nanowires (ESI Fig. S3†).

In Process 4, a drop-cast aqueous solution of cobalt(II) acetate was annealed using the two step process shown in Fig. 4a. The first annealing step was performed for 1 hour in ammonia vapor at $T_{\mathrm{N}}=350-380^{\circ} \mathrm{C}$. This annealing step produced the irregular three dimensional nanocrystallites shown in Fig. 4b. In the second step, ammonia was evacuated from the vacuum chamber, and a 1 hour anneal was performed in a gaseous 1-butanethiol environment at 550-600 ${ }^{\circ} \mathrm{C}\left(T_{\mathrm{S}}\right)$, yielding the nanowires shown in Fig. 4c.

(a)

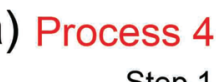

Step 1

\section{Step 2}
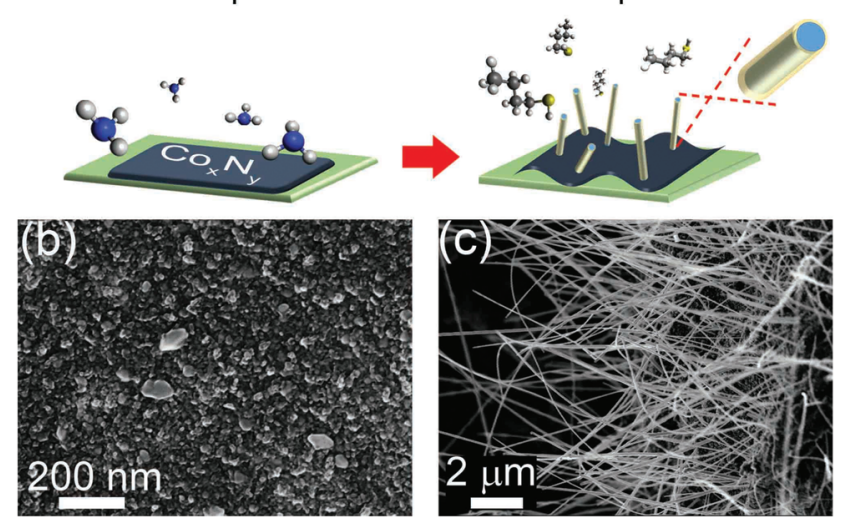

Fig. 4 (a) Schematic of Process 4, in which annealing of a dropcast cobalt salt solution in gaseous ammonia at $T_{\mathrm{N}}=350-380{ }^{\circ} \mathrm{C}$ (Step 1) is followed by annealing in butanethiol at $T_{\mathrm{S}}=550-600{ }^{\circ} \mathrm{C}$ (Step 2). SEM images of the growth product (b) following Step 1, and (c) following Step 2. 
Both the order of $\mathrm{N}$ and S supply and the chosen annealing temperatures were found to be critical to the formation of nanowires. The regimes used in Processes 1-4 were developed in light of previous work on temperature-dependant transformations of cobalt (and other metal) compounds. Decomposition of metal(II) salts in the presence of amines has been shown to form metal nitride species below $300{ }^{\circ} \mathrm{C}^{32-34}$ Under high vacuum conditions or in an inert gas, the metal nitrides decompose upon further heating to $500-570{ }^{\circ} \mathrm{C}$ to form the metal and eliminate nitrogen. ${ }^{35,36}$ Thus, in our technique, nanowire growth proceeds via an intermediate metal nitride phase. Subsequent reduction of the metal nitride to the metallic form in the presence of thiol at $550-600{ }^{\circ} \mathrm{C}$ gives rise to the growth anisotropy responsible for the formation of nanowires.

The growth of one-dimensional structures from metals possessing cubic crystal structure can be understood in the context of symmetry-breaking nanostructure growth. ${ }^{37}$ We propose that the role of sulfur is to passivate nanowire growth in the radial direction. Sulfur (and thiol groups more generally) are well known passivating agents for the growth of metal and semiconductor nanostructures. Such passivation techniques have been widely employed to engineer anisotropic nanocrystal and nanowire growth in the solution phase, ${ }^{37,38}$ but have only recently been extended into the vapor phase, with sulfur being used as a surfactant in chemical vapor deposition (CVD) of GaSb nanowires. ${ }^{39}$ Precursor-bound coordinating ligands can also act as surfactants to direct the CVD growth of gold nanowires and nanoplates. ${ }^{40}$ The current results show that sulfur can be used as both a reducing and structure-directing agent in vapor phase nanowire growth.

Having established the necessary conditions for nanowire growth, we demonstrate the utility of the process to synthesize binary alloy nanowires. In addition to single crystalline Ni and Co nanowires, NiCo, CoFe and FeNi alloy nanowires have been prepared (Fig. 5). Fig. 5a shows Ni nanowires grown using Process 1 in which a dropcast aqueous solution containing $\mathrm{Ni}$ (II) acetate and L-cysteine was annealed in high vacuum at 550-600 ${ }^{\circ} \mathrm{C}$. Fig. 5b shows Ni nanowire growth by Process 3 , in which a dropcast solution containing $\mathrm{Ni}$ (II) acetate and serine is annealed in 1-butanethiol at 550-600 ${ }^{\circ} \mathrm{C}$. NiCo, FeNi and FeCo alloy nanowires were also synthesized using Process 3 with the precursor molar ratios listed in Table 1. Typical morphologies of NiCo, FeNi and FeCo nanowires are shown in Fig. 5c-e respectively. In all cases, the highest nanowire concentration occurs at sites where the film has undergone delamination. SEM and SAED characterization of single crystalline NiCo nanowires grown by Process 1 at $430^{\circ} \mathrm{C}$ is provided in Fig. $6 a$ and $b$ respectively. The SAED pattern can be
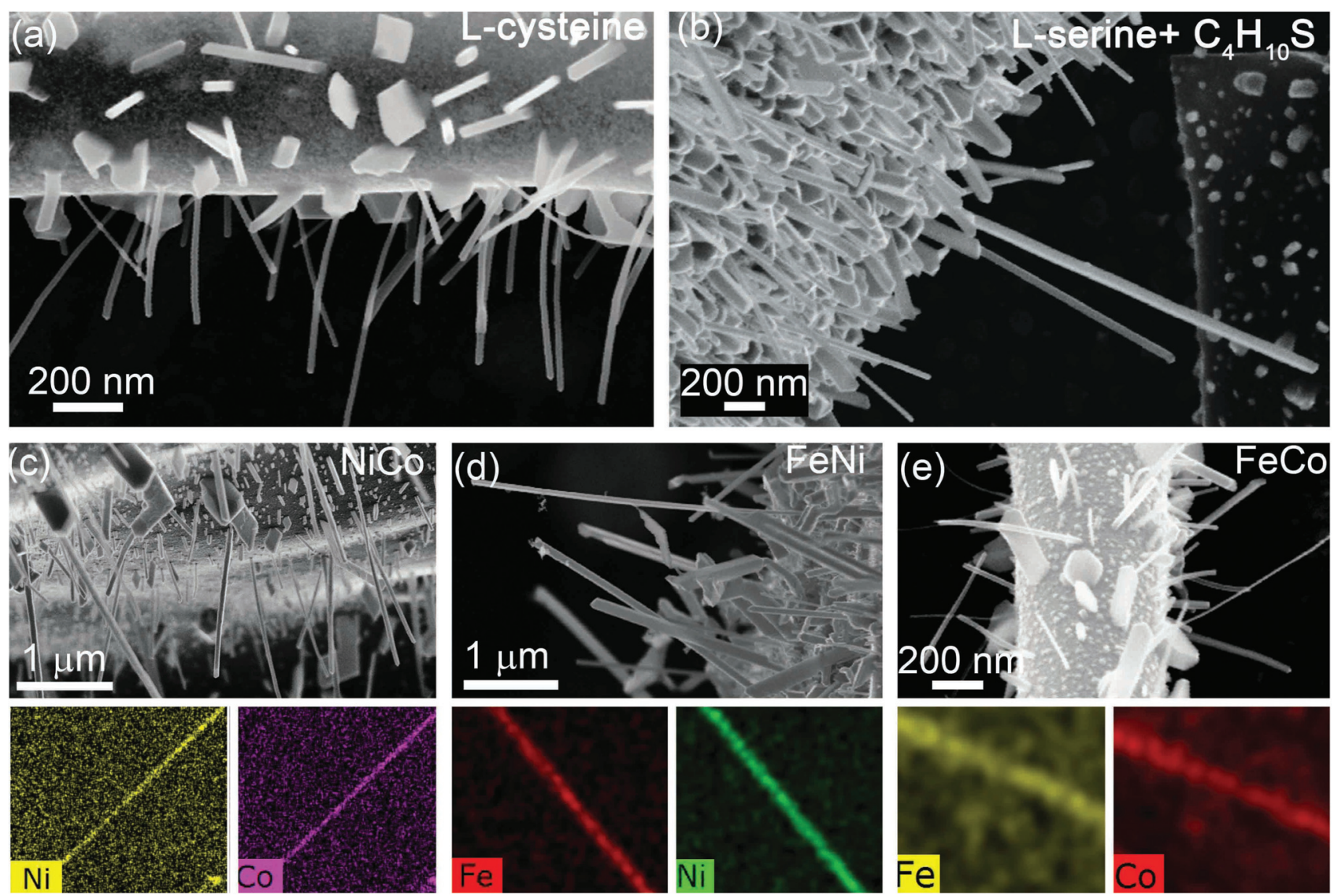

Fig. 5 (a) Ni nanowires grown by Process 1. (b) Ni nanowires grown by Process 3. (c)-(e) NiCo, FeNi and FeCo alloy nanowire growth from Process 3, with corresponding EDS maps. 


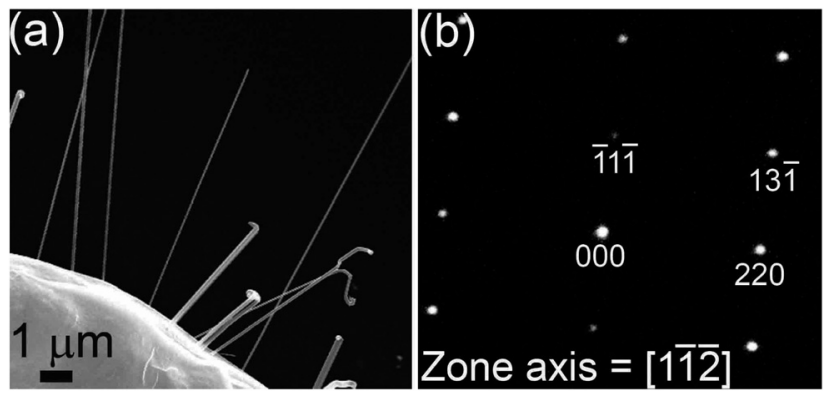

Fig. 6 (a) SEM image of NiCo alloy NWs grown by Process 1. (b) SAED pattern of a NiCo nanowire, which can be indexed to a FCC crystal structure.

indexed to the FCC phase of NiCo. The composition of these binary alloy nanowires can be tuned by suitable adjustment of the precursor molar ratios.

\section{Conclusions}

These results represent the first demonstration of high quality metal nanowire fabrication with a wide range of compositions by a template-free technique. The nanowire growth proceeds through an amine-promoted metal nitride intermediate, which is subsequently reduced to the metallic phase in the presence of thiol. The thiol acts as both a reducing agent and a uniaxial structure-directing agent. The amine and thiol functionalities can be introduced as solution-phase precursors or in vapor phase but importantly, both are necessary for nanowire growth. The versatility of the method has been demonstrated by growth of uniform single-crystalline metal and alloy nanowires with a range of compositions (Co, Ni, NiCo, CoFe, and $\mathrm{NiFe}$ ) on metal as well as semiconducting surfaces.

\section{Methods}

\section{Process 1}

$\mathrm{Si}$ and $\mathrm{SiO}_{2}$ wafers with a $100 \mathrm{~nm}$ thermal oxide layer were cut to $5 \mathrm{~mm} \times 5 \mathrm{~mm}$ substrates and sonicated for 10 minutes each in acetone, isopronyl, and MilliQ water. Cobalt acetate $(0.2 \mathrm{~g}$, cobalt(II) acetate tetrahydrate 98\% Sigma Aldrich) and L-cysteine $(0.3 \mathrm{~g}, 97 \%$ Sigma Aldrich) were dissolved in $24 \mathrm{~mL}$ DI water in atmosphere with no further purification procedures. The solution was sonicated for 5 minutes and then drop-cast onto the substrate at $100{ }^{\circ} \mathrm{C}$. The substrate was loaded into a high vacuum chamber and heated in a single step to the growth temperature (550-600 $\left.{ }^{\circ} \mathrm{C}\right)$. Growth was performed in high vacuum $\left(10^{-5}\right.$ mbar) for 1 hour, before the sample was cooled to room temperature and removed for characterization.

\section{Process 2}

The substrate was heated to $145{ }^{\circ} \mathrm{C}$ and the chamber filled with $\mathrm{Co}(\mathrm{CO})_{3} \mathrm{NO}$ vapour to a pressure of 0.27 mbar. After
20 minutes, $\mathrm{Co}(\mathrm{CO})_{3} \mathrm{NO}$ was exhausted, and both ammonia $(0.7 \mathrm{sccm})$ and butanethiol were introduced until the total pressure reached 0.13 mbar. The substrate was heated to $60^{\circ} \mathrm{C}$ in the butanethiol/ammonia gas mixture for 1 hour before the gases were exhausted and the substrate cooled to room temperature.

\section{Process 3}

Co(II) acetate $(0.02 \mathrm{~g}$, cobalt(II) acetate tetrahydrate $98.0 \%$ Sigma Aldrich) and serine (0.04 g, 99.0\% Sigma Aldrich) were dissolved in $2 \mathrm{~mL}$ ethanol. The substrate was loaded into the chamber and evacuated to 0.03 mbar. Butanethiol was then introduced into the chamber to a pressure of 0.3 mbar. The sample was then heated to $550{ }^{\circ} \mathrm{C}$ in butanethiol at a rate of $30{ }^{\circ} \mathrm{C}$ per minute, held at $550{ }^{\circ} \mathrm{C}$ for 1 hour, then cooled to room temperature and removed for characterization.

\section{Process 4}

Cobalt acetate $(0.2 \mathrm{~g}$, cobalt(II) acetate tetrahydrate $98.0 \%$ Sigma Aldrich) was dissolved in $24 \mathrm{~mL}$ DI water in atmosphere with no further purification procedures. The solution was drop-cast onto a substrate held at $100{ }^{\circ} \mathrm{C}$. After loading the substrate into the growth chamber, it was evacuated to $0.03 \mathrm{mbar}$ and ammonia was introduced at a flow rate of 0.5 sccm. The substrate was heated to $350{ }^{\circ} \mathrm{C}$ as the pressure was increased to 0.4 mbar $\mathrm{NH}_{3}$. After 60 minutes, $\mathrm{NH}_{3}$ was exhausted, butanethiol was introduced at $0.5 \mathrm{sccm}$ up to a total system pressure of $0.4 \mathrm{mbar}$, and the substrate was annealed at $550-600{ }^{\circ} \mathrm{C}$ for 1 hour.

\section{Alloy nanowire growth}

\section{NiCo}

Process 1. Cobalt acetate (0.03 g, cobalt(II) acetate tetrahydrate 98\% Sigma Aldrich), nickel acetate (0.03 g, nickel(II) acetate tetrahydrate $99.998 \%$ Sigma Aldrich) and L-cysteine (0.1 g, 97\% Sigma Aldrich) were dissolved in $8 \mathrm{~mL}$ DI water in atmosphere with no further purification procedures. The solution was sonicated for 5 minutes and then drop-cast onto the substrate at $100{ }^{\circ} \mathrm{C}$. The substrate was loaded into a high vacuum chamber and heated in a single step to the growth temperature $\left(420-470{ }^{\circ} \mathrm{C}\right)$. Growth was performed in high vacuum $\left(10^{-5}\right.$ mbar $)$ for 1 hour, before the sample was cooled to room temperature and removed for characterization.

Process 3. Ni(II) acetate (0.1 g, nickel(II) acetate tetrahydrate 99.998\% Sigma Aldrich), Co(II) acetate ( $0.1 \mathrm{~g}$, cobalt(II) acetate tetrahydrate $98.0 \%$ Sigma Aldrich) and serine (0.3 g, 99.0\% Sigma Aldrich) were dissolved in $24 \mathrm{~mL}$ DI $\mathrm{H}_{2} \mathrm{O}$. The substrate was loaded into the chamber and evacuated to 0.03 mbar. Butanethiol was then introduced into the chamber to a pressure of 0.3 mbar ( 0.23 Torr). The sample was then heated to $550{ }^{\circ} \mathrm{C}$ in butanethiol at a rate of $30^{\circ} \mathrm{C}$ per minute, held at $550{ }^{\circ} \mathrm{C}$ for 1 hour, then cooled to room temperature and removed for characterization.

\section{FeCo}

Process 3. $\mathrm{Fe}$ (II) acetate (0.063 g, iron(II) acetate 95\% Sigma Aldrich), Co(II) acetate ( $0.1 \mathrm{~g}$, cobalt(II) acetate tetrahydrate 
98.0\% Sigma Aldrich) and serine (0.3 g, 99.0\% Sigma Aldrich) were dissolved in $24 \mathrm{~mL} \mathrm{DI} \mathrm{H}_{2} \mathrm{O}$. The substrate was loaded into the chamber and evacuated to 0.03 mbar. Butanethiol was then introduced into the chamber to a pressure of 0.3. The sample was then heated to $550{ }^{\circ} \mathrm{C}$ in butanethiol at a rate of $30{ }^{\circ} \mathrm{C}$ per minute, held at $550{ }^{\circ} \mathrm{C}$ for 1 hour, then cooled to room temperature and removed for characterization.

\section{FeNi}

Process 3. Fe(II) acetate (0.063 g, iron(II) acetate 95\% Sigma Aldrich), Ni(II) acetate (0.1 g, nickel(II) acetate tetrahydrate 99.998\% Sigma Aldrich) and serine (0.3 g, 99.0\% Sigma Aldrich) were dissolved in $24 \mathrm{~mL}$ DI $\mathrm{H}_{2} \mathrm{O}$. The substrate was loaded into the chamber and evacuated to 0.03 mbar. Butanethiol was then introduced into the chamber to a pressure of 0.3 mbar. The sample was then heated to $55^{\circ} \mathrm{C}$ in butanethiol at a rate of $3{ }^{\circ} \mathrm{C}$ per minute, held at $55{ }^{\circ} \mathrm{C}$ for 1 hour, then cooled to room temperature and removed for characterization.

\section{SEM and TEM characterization}

SEM imaging was performed at $5 \mathrm{kV}$ in a Zeiss Supra 55VP directly following growth. For TEM analysis an identical solution containing (L-cysteine and Co(II) acetate tetrahydrate) was prepared and dropcast onto a $300 \mu \mathrm{m} \mathrm{Au}$ mesh and heated under identical growth conditions. TEM analysis was carried out on either a FEI Tecnai T20 or JEM-2200FS FEG.

\section{Acknowledgements}

The authors thank S. Lifshitz, M. T. Westerhausen, and C. Zachreson for discussions. The work was supported in part by the Australian Research Council (project no. DP140102721) and FEI Company. A.A.M. is the recipient of a John Stocker Postgraduate Scholarship from the Science and Industry Endowment Fund. I.A. is the recipient of an Australian Research Council Discovery Early Career Research Award (project no. DE130100592).

\section{References}

1 S. S. P. Parkin, M. Hayashi and L. Thomas, Magnetic Domain-Wall Racetrack Memory, Science, 2008, 320, 190.

2 M. M. Maqableh, X. Huang, S.-Y. Sung, K. S. M. Reddy, G. Norby, R. H. Victora and B. J. H. Stadler, Low-Resistivity 10 Nm Diameter Magnetic Sensors, Nano Lett., 2012, 12, 4102.

3 W. Gao, S. Sattayasamitsathit, K. M. Manesh, D. Weihs and J. Wang, Magnetically Powered Flexible Metal Nanowire Motors, J. Am. Chem. Soc., 2010, 132, 14403.

4 X. Kou, X. Fan, R. K. Dumas, Q. Lu, Y. Zhang, H. Zhu, X. Zhang, K. Liu and J. Q. Xiao, Memory Effect in Magnetic Nanowire Arrays, Adv. Mater., 2011, 23, 1393.

5 H.-T. Huang, T.-R. Ger, Y.-H. Lin and Z.-H. Wei, Single Cell Detection Using a Magnetic Zigzag Nanowire Biosensor, Lab Chip, 2013, 13, 3098.
6 X. Huang, Y. Li, Y. Li, H. Zhou, X. Duan and Y. Huang, Synthesis of Ptpd Bimetal Nanocrystals with Controllable Shape, Composition, and Their Tunable Catalytic Properties, Nano Lett., 2012, 12, 4265.

7 N. Gao, H. Wang and E.-H. Yang, An Experimental Study on Ferromagnetic Nickel Nanowires Functionalized with Antibodies for Cell Separation, Nanotechnology, 2010, 21, 105107.

8 K. M. Pondman, N. D. Bunt, A. W. Maijenburg, R. J. A. van Wezel, U. Kishore, L. Abelmann, J. E. ten Elshof and B. ten Haken, Magnetic Drug Delivery with Fepd Nano Wires, J. Magn. Magn. Mater., 2015, 380, 299.

9 F. Garcia-Sanchez, H. Szambolics, A. P. Mihai, L. Vila, A. Marty, J. P. Attane, J. C. Toussaint and L. D. Buda-Prejbeanu, Effect of Crystalline Defects on Domain Wall Motion under Field and Current in Nanowires with Perpendicular Magnetization, Phys. Rev. B: Condens. Matter, 2010, 81, 134408.

10 J. Zhang, X. Wang, X. Peng and L. Zhang, Fabrication, Morphology and Structural Characterization of Ordered SingleCrystal Ag Nanowires, Appl. Phys. A: Mater. Sci. Process., 2002, 75, 485.

11 M. L. Tian, J. U. Wang, J. Kurtz, T. E. Mallouk and M. H. W. Chan, Electrochemical Growth of Single-Crystal Metal Nanowires Via a Two-Dimensional Nucleation and Growth Mechanism, Nano Lett., 2003, 3, 919.

12 H. Pan, B. H. Liu, J. B. Yi, C. Poh, S. Lim, J. Ding, Y. P. Feng, C. H. A. Huan and J. Y. Lin, Growth of Singlecrystalline Ni and Co Nanowires Via Electrochemical Deposition and Their Magnetic Properties, J. Phys. Chem. B, 2005, 109, 3094.

13 L. G. Vivas, Y. P. Ivanov, D. G. Trabada, M. P. Proenca, O. Chubykalo-Fesenko and M. Vazquez, Magnetic Properties of Co Nanopillar Arrays Prepared from Alumina Templates, Nanotechnology, 2013, 24, 105703.

14 T. Thurn-Albrecht, J. Schotter, C. A. Kastle, N. Emley, T. Shibauchi, L. Krusin-Elbaum, K. Guarini, C. T. Black, M. T. Tuominen and T. P. Russell, Ultrahigh-Density Nanowire Arrays Grown in Self-Assembled Diblock Copolymer Templates, Science, 2000, 290, 2126.

15 L. Vila, P. Vincent, L. Dauginet-De Pra, G. Pirio, E. Minoux, L. Gangloff, S. Demoustier-Champagne, N. Sarazin, E. Ferain, R. Legras, L. Piraux and P. Legagneux, Growth and Field-Emission Properties of Vertically Aligned Cobalt Nanowire Arrays, Nano Lett., 2004, 4, 521.

16 J. Qin, J. Nogues, M. Mikhaylova, A. Roig, J. S. Munoz and M. Muhammed, Differences in the Magnetic Properties of Co, Fe, and Ni 250-300 Nm Wide Nanowires Electrodeposited in Amorphous Anodized Alumina Templates, Chem. Mater., 2005, 17, 1829.

17 X. Huang, L. Li, X. Luo, X. Zhu and G. Li, OrientationControlled Synthesis and Ferromagnetism of Single Crystalline Co Nanowire Arrays, J. Phys. Chem. C, 2008, 112, 1468.

18 A. Cortes, G. Riveros, J. L. Palma, J. C. Denardin, R. E. Marotti, E. A. Dalchiele and H. Gomez, Single-Crystal 
Growth of Nickel Nanowires: Influence of Deposition Conditions on Structural and Magnetic Properties, J. Nanosci. Nanotechnol., 2009, 9, 1992.

19 Y. P. Ivanov, L. G. Vivas, A. Asenjo, A. Chuvilin, O. Chubykalo-Fesenko and M. Vazquez, Magnetic Structure of a Single-Crystal Hcp Electrodeposited Cobalt Nanowire, EPL, 2013, 102, 17009.

20 M. Lai and D. J. Riley, Templated Electrosynthesis of Nanomaterials and Porous Structures, J. Colloid Interface Sci., 2008, 323, 203.

21 K. T. Chan, J. J. Kan, C. Doran, O. Y. Lu, D. J. Smith and E. E. Fullerton, Oriented Growth of Single-Crystal Ni Nanowires onto Amorphous $\mathrm{Sio}_{2}$, Nano Lett., 2010, 10, 5070.

22 N. Bagkar, K. Seo, H. Yoon, J. In, Y. Jo and B. Kim, Vertically Aligned Single-Crystalline Ferromagnetic Ni3co Nanowires, Chem. Mater., 2010, 22, 1831.

23 N. Liakakos, T. Blon, C. Achkar, V. Vilar, B. Cormary, R. P. Tan, O. Benamara, G. Chaboussant, F. Ott, B. WarotFonrose, E. Snoeck, B. Chaudret, K. Soulantica and M. Respaud, Solution Epitaxial Growth of Cobalt Nanowires on Crystalline Substrates for Data Storage Densities Beyond 1 Tbit/In ${ }^{2}$, Nano Lett., 2014, 14, 3481.

24 S.-i. Kim, H. Yoon, H. Lee, S. Lee, Y. Jo, S. Lee, J. Choo and B. Kim, Epitaxy-Driven Vertical Growth of Single-Crystalline Cobalt Nanowire Arrays by Chemical Vapor Deposition, J. Mater. Chem. C, 2015, 3, 100.

25 Y. Soumare, C. Garcia, T. Maurer, G. Chaboussant, F. Ott, F. Fievet, J. Y. Piquemal and G. Viau, Kinetically Controlled Synthesis of Hexagonally Close-Packed Cobalt Nanorods with High Magnetic Coercivity, Adv. Funct. Mater., 2009, 19, 1971.

26 K. Gandha, K. Elkins, N. Poudyal, X. B. Liu and J. P. Liu, High Energy Product Developed from Cobalt Nanowires, Sci. Rep., 2014, 4, 5345.

27 F. Dumestre, B. Chaudret, C. Amiens, B. Chaudret, M. C. Fromen, M. J. Casanove, P. Renaud and P. Zurcher, Shape Control of Thermodynamically Stable Cobalt Nanorods through Organometallic Chemistry, Angew. Chem., Int. Ed., 2002, 41, 4286.

28 X.-L. Liu and Y.-J. Zhu, A Precursor Nanowire Templated Route to Cds Nanowires, Mater. Lett., 2009, 63, 1085.

29 Y. O. Chen and Y. A. Cheng, in Metallurgical Transactions B, 2008, vol. 9B, p. 61.
30 Z. L. Zhang, Surface Effects in the Energy Loss near Edge Structure of Different Cobalt Oxides, Ultramicroscopy, 2007, 107, 598.

31 Z. Wang, L. Pan, H. Hu and S. Zhao, CrystEngComm, 2010, $12,1899$.

32 J. Zheng, R. Yang, W. Chen, L. Xie, X. Li and C. Chen, Iron Nitride Thin Films Deposited by Chloride Assisted Plasma Enhanced Chemical Vapour Deposition: Facile Stoichiometry Control and Mechanism Study, J. Phys. D: Appl. Phys., 2009, 42, 185209.

33 A. N. Cloud, L. M. Davis, G. S. Girolami and J. R. Abelson, Low-temperature CVD of iron, cobalt and nickel nitride thin films from bis[di(tertbutyl)amido]metal(II) precursors and ammonia, J. Vac. Sci. Technol., A, 2014, 32, 020606.

34 M. B. Lourenco, M. D. Carvalho, P. Fonseca, T. Gasche, G. Evans, M. Godinho and M. M. Cruz, Stability and Magnetic Properties of Cobalt Nitrides, J. Alloys Compd., 2014, 612, 176.

35 L. Maya, M. Paranthaman, J. R. Thompson, T. Thundat and R. J. Stevenson, Ferromagnetic Nanocomposite Films of Cobalt in a Ceramic Matrix Formed by Thermal Decomposition of Cobalt Nitride, Con, Precursor, J. Appl. Phys., 1996, 79, 7905.

36 M. Widenmeyer, T. C. Hansen, E. Meissner and R. Niewa, Formation and Decomposition of Iron Nitrides Observed by in Situ Powder Neutron Diffraction and Thermal Analysis, Z. Anorg. Allg. Chem., 2014, 640, 1265.

37 B. Viswanath, P. Kundu, A. HaIder and N. Ravishankar, Mechanistic Aspects of Shape Selection and Symmetry Breaking During Nanostructure Growth by Wet Chemical Methods, J. Phys. Chem. C, 2009, 113, 16866.

38 J. Chen, B. J. Wiley and Y. Xia, One-Dimensional Nanostructures of Metals: Large-Scale Synthesis and Some Potential Applications, Langmuir, 2007, 23, 4120.

39 Z.-x. Yang, N. Han, M. Fang, H. Lin, H.-Y. Cheung, S. Yip, E.-J. Wang, T. Hung, C.-Y. Wong and J. C. Ho, SurfactantAssisted Chemical Vapour Deposition of High-Performance Small-Diameter Gasb Nanowires, Nat. Commun., 2014, 5, 5249.

40 M. B. E. Griffiths, S. E. Koponen, D. J. Mandia, J. F. McLeod, J. P. Coyle, J. J. Sims, J. B. Giorgi, E. R. Sirianni, G. P. A. Yap and S. T. Barry, Surfactant Directed Growth of Gold Metal Nanoplates by Chemical Vapor Deposition, Chem. Mater., 2015, 27, 6116. 